\title{
Dynamic characteristics of spatial mechanisms coding contour structures
}

\author{
BIRGITTA DRESP \\ Laboratoire de Systèmes Biomécaniques I.M.F., U.M.R. 7507-C.N.R.S., Université Louis Pasteur \\ Strasbourg, France
}

Received 20 February 1998; accepted 5 August 1998

\begin{abstract}
Psychophysical thresholds for the detection of luminance targets improve significantly when the targets are presented in a specific context of spatially separated, collinear inducing stimuli defining visual contours. This phenomenon is generally referred to as a special case of detection facilitation called spatial facilitation. Spatial facilitation has been observed with luminance-defined, achromatic stimuli on achromatic backgrounds as well as with targets and inducers defined by colour contrast. This paper reviews psychophysical results from detection experiments with human observers showing the conditions under which spatially separated contour inducers facilitate the detection of simultaneously presented target stimuli. The findings point towards two types of spatial mechanisms: (i) Short-range mechanisms that are sensitive to narrowly spaced stimuli of small size and, at distinct target locations, selective to the contrast polarity of targets and inducers. (ii) Long-range mechanisms that are triggered by longer stimuli, generate facilitation across wider spatial gaps between targets and inducers, and are insensitive to their contrast polarity. Spatial facilitation with chromatic stimuli requires a longer inducer exposure than spatial facilitation with achromatic stimuli, which is already fully effective at inducer exposures of $30 \mathrm{~ms}$. This difference in temporal dynamics indicates some functional segregation between mechanisms for colour and luminance contrast in spatial coding. In general, spatially induced detection facilitation can to a large extent be explained by mechanisms involving from-short-to-long-range interactions between cortical detectors.
\end{abstract}

\section{INTRODUCTION}

Psychophysical experiments on detection facilitation with collinear targets and inducers, now called spatial facilitation (Yu and Levi, 1997a), have generated a coherent body of data supporting hypotheses about visual mechanisms of spatial grouping and the early processing of object contours and illusory contours (Dresp and Bonnet, 1991, 1993; Dresp, 1993; Polat and Sagi, 1993, 1994a, b; Dresp and Bonnet, 1995; Kapadia et al., 1995; Dresp and Grossberg, 1997; Yu and Levi, 1997a; Wehrhahn and Dresp, 1998). The general finding is that the 
visual detection of a target stimulus can be facilitated, suppressed, or remain unaltered by nearby context stimuli depending on their spatial location, orientation, contrast intensity, contrast polarity, or colour (see Fig. 1 for an illustration). These facilitatory or suppressive interactions between features are supposed to reveal dynamic characteristics of mechanisms underlying the perception of visual structure. This interpretation of the psychophysical data takes into account the neurophysiological evidence that the response characteristics of visual cortical cells change with the context in which the trigger-stimuli are presented (e.g. Gilbert and Wiesel, 1990). In the following paragraphs, some of the dynamic characteristic of spatial facilitation and visual grouping will be reviewed. The relevance of spatial facilitation effects with regard to hypotheses about neurophysiological mechanisms is discussed, and it is shown how the spatial, structural, and temporal conditions governing the visual integration of stimuli presented within or without a context of contour structures can be related to the functional characteristics of, presumably hierarchically organized, neural mechanisms operating within short- and long-range spatial scales.

\section{SPATIAL FACILITATION AND THE DYNAMICS OF EARLY VISUAL GROUPING}

Psychophysical experiments demonstrating detection facilitation or suppression induced by spatially separated visual stimuli selectively probe the multiple levels of visual processing that influence the formation of object contours, perceptual boundaries, and virtual or illusory contours. They allow to identify the spatial, structural, and temporal stimulus parameters that are relevant for the grouping of visual information, and lead to increasingly refined hypotheses regarding the neurophysiological mechanisms underlying the perception of structure, unity, and form in pictorial scenes.

\section{Psychophysical measures of spatial facilitation}

Psychophysical experiments on spatial facilitation use luminance detection tasks with briefly presented visual targets, usually lines, small squares, or dots. Generally, two-alternative forced-choice procedures are run, where the observer has to decide whether the target was presented on the left or the right side of the computerscreen (spatial forced choice), or whether it was presented in the first or in the second of two successive temporal intervals (temporal forced choice). Different luminance levels of the target are presented, either within an adaptive staircase procedure, or according to the method of constant stimuli. Individual detection thresholds, corresponding to the luminance level at which the observer correctly detects the target in $75 \%$ of the trials, are computed through statistical inter- or extrapolation procedures.

The effect of a configurational stimulus context on the detection threshold of the target is evaluated by comparing situations where the target is presented within a 

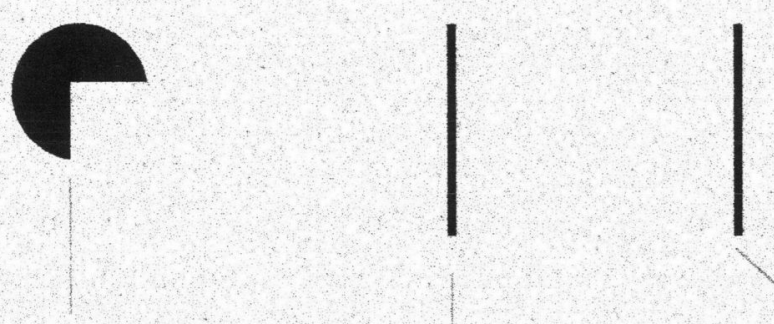

A

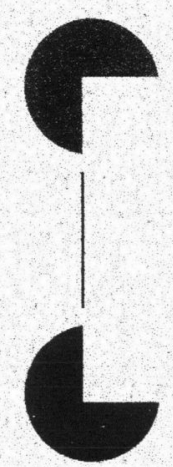

D
B

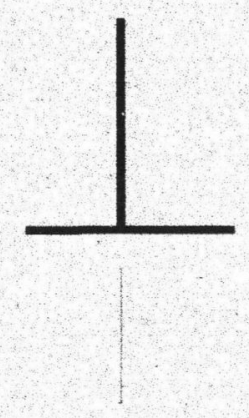

E
C

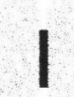

I

F

Figure 1. Spatial facilitation with targets and inducers defining contour structures is described by the fact that a structured context lowers the psychophysical detection threshold for a contrast target. Examples of structured contour contexts which either facilitate or suppress the detectability of line targets (represented by the thinner lines here) are shown in this figure (for details about these effects and references, see text). Contour structures with collinear edges generally facilitate the detection of line targets presented within the gap between edges (A), and so do simple line structures (B and C). Chromatic contour structures only facilitate the detection of chromatic targets (D), not achromatic ones. Achromatic contour structures only facilitate the detection of achromatic targets. Contour structures suppress target detectability when an orthogonal contour is presented between collinear targets and inducers (E), or when the contrast polarity of the contour structure is not homogenous on a given orientation axis $(\mathrm{F})$. 
context to control situations where it is flashed without the context under otherwise exactly the same conditions. Spatial facilitation occurs when a stimulus context lowers the detection threshold compared to a control condition without context. In this case, we may assume that the target and the context generate cooperative input that triggers a spatial mechanism coding visual structure. In some cases, context stimuli lead to an increase in detection thresholds. In this case we may assume that the suppressive effect reflects the fact that target and context generate competitive input that inhibits a spatial mechanism coding structure. When context stimuli do not affect detection thresholds of the target compared to the control situation, we may assume that target and context stimulate independent mechanisms the combination of which is unlikely to generate representations of visual structure. Spatial facilitation as it is defined above only occurs with specific target/context combinations. One of the critical determinants of spatial facilitation has been found to be stimulus alignment, or collinearity of target and context.

\section{Collinearity of targets and inducers}

In a perceptual environment of discrete visual events, the spatial alignment of points or fragments is directly related to the likelihood that these points or fragments belong to the same object contour. Finely tuned mechanisms with a high sensitivity to alignment are necessary for the detection of continuity in stimuli defined by discrete points aligned in space, and psychophysical evidence for their existence comes from a whole body of data on visual hyperacuity showing that thresholds for the smallest detectable misalignment of abutting lines are measurable in seconds of arc, and can be up to a factor of 10 smaller than the closest spacing of foveal cones (Westheimer, 1979).

The integration of object contours across space, including the formation of illusory contours, is likely to be determined by visual mechanisms that allow for discontinuities, or spatial gaps, in the stimulus input. The role of these mechanisms would be to recover the missing information via some kind of bridging operation. Field et al. (1993), for example, have shown that human observers are capable to detect a continuous path defined by aligned contour elements within a field of randomly oriented elements even when the spacing of the aligned features is considerably larger than the size of any of the individual elements. Alignment, or collinearity, thus produces what the authors call a local 'association field' which is processed by the visual system on the basis of the Gestalt law of 'good continuation'. Furthermore, perceptual closure appears to strengthen the effect of alignment on contour identification when the individual segments form curved Gestaiten (see, for example, Kovács and Julesz, 1993).

Interactions between orientation selective cortical neurons account for the way in which the grouping of spatially separated contour segments is achieved by the visual system. Kapadia et al. (1995) have measured the effect of collinearity on both human contrast thresholds and of superficial layer complex cells in monkey V1. It was found that a human observer's line contrast detection is significantly 
improved by the presence of collinear, suprathreshold context lines. The firing rates of complex cortical cells in the monkey showed the same contextual dependency on the relative location and orientation of the lines. Spatial facilitation with contour inducers has also been found to be influenced by the alignment of targets and inducers. While contrast thresholds for a circular target are facilitated by a collinear line inducer, it has been found that the facilitatory effect diappears when an orthogonal context element, destroying perceptual alignment, is presented between the target and the inducer (Dresp, 1991, 1993).

However, other spatial facilitation experiments have shown that the collinearity of targets and inducers is, although in most cases efficient, not always required for obtaining facilitatory effects of spatial inducers on target detection. Dresp and Grossberg (1995) observed detection facilitation of line targets inserted between spatially separated, perpendicular inducing lines. In this case, the target was aligned with the ends of the inducers, only. This result indicates that targets and inducers the ends of which can be connected by a straight line do not have to share the same orientation to produce spatial facilitation. Moreover, target-inducer collinearity is not a necessary condition for spatial facilitation with line targets and square shaped inducers (Yu and Levi, 1997a). Placing the squares at the two lateral flanks of the line target produces detection facilitation within precise spatial limits situated between 6 and 22 arcmin of lateral distance between target line and inducers. This result suggests that in some cases spatial facilitation might reflect the output of a circular, or elliptic spatial filter with antagonistic surrounding regions, identified with the typical functional characteristics of retinal ganglion cells or cortical detectors with end-stopped receptive field profiles (Hubel and Wiesel, 1965). However, it appears that a more complex picture of the conditions determining spatial facilitation emerges when length and spatial separation of targets and inducers are varied.

\section{Length and spatial separation of targets and inducers}

The likelihood that spatially aligned stimulus segments belong to the same object contour should be the higher the smaller the spatial separation of the individual segments. Zucker and Davis (1988), for example, proposed that the likelihood of an array of collinear dots to be grouped into a continuous contour by the visual system is directly determined by some size/spacing ratio which predicts perceived contour strength in a psychophysical task. In spatial facilitation studies and related investigations, it has been shown that one of the striking consequences of variations in target-inducer spacing is that the effects of collinearity on contrast thresholds change, or are found to be completely reversed. With aligned Gabor patches as target/inducer configurations (Polat and Sagi, 1993, 1994a), the at first suppressive effect of collinear high-contrast inducers on contrast thresholds of the target reverses into a facilitatory effect at some critical target-inducer separation. This sudden facilitation then diminishes again when the spacing of target and inducers increases further (Polat and Sagi, 1993, 1994a). Moreover, the dependency 
of contrast thresholds on alignment and spatial separation was found to be invariant across different global orientations, including curves (Polat and Sagi, 1994b). In configurations with edge-like spatial inducers, increasing the gap between two collinar inducers decreases facilitatory effects on the detection of a small light increment presented within that gap (Dresp and Bonnet, 1991, 1993). Detection facilitation disappears completely at a target-inducer separation of about $1.75 \mathrm{deg}$ of visual angle with edge-like inducers of constant size. Furthermore, suppressive effects reflected through local threshoid elevations observed near the lateral flanks of the inducers, decrease with increasing inducer spacing (Dresp and Bonnet, 1993).

The apparent complexity of the effects that target-inducer separation and length seem to engender in spatial facilitation is one of the key issues in current studies. While Yu and Levi's (1997a) data show spatial facilitation with short targets and inducers within spatial limits up to 20 arcmin only, other findings with much longer targets and inducing lines indicate that spatial facilitation occurs within larger spatial scales as well (Dresp and Grossberg, 1997; Wehrhahn and Dresp, 1998). Here again, the conclusions are not straightforward. In fact, it appears that the contrast intensity and relative contrast polarity of targets and inducers determine whether detection facilitation occurs for a given inducer and target length, or spatial separation.

\section{Inducer contrast and contrast polarity}

In classic studies investigating the effects of contrast stimuli, also sometimes referred to as 'pedestal' stimuli, on the detectability of nearby contrast targets, it has been shown that high-contrast inducers usually mask nearby stimuli, while low-contrast inducers most often facilitate the contrast detection of nearby targets (e.g. Foley and Legge, 1981; Morgan and Dresp, 1995). More recent work on spatial facilitation with collinear targets and inducers, however, has shown that these pedestal effects may disappear, or be reversed, depending on the contrast polarity and the spacing of contrast targets and inducers. When the target and the inducer have the same contrast polarity, some of the results are consistent with classic pedestal effects, provided the target-inducer separation is small (e.g. Morgan and Dresp, 1995; Wehrhahn and Dresp, 1998). Under these conditions, detection facilitation is found to be strongest with low-contrast inducers, and decreases when the inducer contrast increases. This observation can be regarded as a predictable consequence of Weber's law where low contrast inducers enhance the perceived contrast of the target via subthreshold summation and high contrast inducers suppress the perceived contrast of the target via masking. However, with targets and inducers of small size ( 3 arcmin long and wide) and opposite contrast polarity, detection facilitation has been reported when the spatial gap between target and inducer is not shorter than 3 and not longer than 11 arcmin (Yu and Levi, 1997a).

Furthermore, high contrast inducers masking a target of the same contrast polarity when their spatial separation is relatively small have been found to facilitate detection of the same target when spatial separation is increased further beyond 
some critical limit (Zenger and Sagi, 1996). In fact, with the optimal spatial separation, high contrast inducers have been found to facilitate the detection of targets of either contrast polarity (Zenger and Sagi, 1996; Wehrhahn and Dresp, 1998), whereas low contrast inducers lose their potential to generate detection facilitation. Wehrhahn and Dresp (1998) measured spatial facilitation with line targets as a function of the contrast strength of inducing lines of either contrast polarity. When the target-inducer gap was larger than 20 arcmin, somewhere beyond the critical gap zone described in Yu and Levi's (1997a) experiments, only inducing lines of high contrast produced detection facilitation, regardless of the contrast polarity of the target line. Facilitatory effects disappeared completely with low contrast inducers. This surprising result may reflect the output of a mechanism of contour grouping that is activated only when the decreasing proximity of stimulus fragments is compensated by stronger local contrasts, whichever sign they carry.

Dresp and Grossberg (1997) conducted spatial facilitation studies with edge-like inducers and fractioned line targets collinear with the orientation of the inducing edges. In some conditions, the contrast sign of half the target line was the same as that of the nearest inducer, in others it carried the opposite sign. It was found that, for a given constant spatial separation which was in all cases smaller than 20 arcmin, detection facilitation only occurred when the local contrast polarities of the fractioned target line were the same as those of the nearest inducers. The disposition of the visual system to integrate contour information across relatively small spatial gaps is therefore clearly selective to the contrast sign carried by collinear contour segments. This selectivity depends on the exact size and spatial position of the segments, and the underlying spatial mechanisms appear to produce so-called 'perceptive fields' (Jung and Spillmann, 1970; Yu and Levi, 1997b) which allow to psychophysically identify the size-distant ratio for which a given mechanism is optimally activated. The 'perceptive field' hypothesis finds further support in some brandnew data showing that the spatial limits of contrast detection facilitation correspond to positional acuity thresholds for the same contour stimuli (Dresp et al., 1998). Variations in these thresholds with the size/distance ratio of the stimuli are investigated in current experiments. In summary, it has become clear that different mechanisms, with differential sensitivity to relative stimulus orientation, position, visibility, size, polarity, and colour must operate within the spatial scales in which detection facilitation effects have been observed.

\section{Luminance contrast versus colour contrast}

Psychophysical experiments on spatial facilitation comparing context configurations defined by colour and/or luminance contrast indicate that colour and luminance are likely to be grouped by different mechanisms. Evidence for a functional segregation at some level can be found in the observation that achromatic contour inducers facilitate the detection of achromatic targets with varying luminance, but not the detection of chromatic targets with varying luminance. Chromatic contour inducers facilitate the detection of chromatic targets with varying luminance only 
(Dresp and Grossberg, 1998). Furthermore, it appears that the timecourse of achromatic spatial facilitation is significantly shorter than that of chromatic facilitation (Dresp and Grossberg, 1998), an observation that is consistent with findings reported by Leonards and Singer (1998) with temporally induced texture segmentation. Leonards and Singer conclude that orientation cues supporting texture segmentation are processed by two mechanisms with differential sensitivity for colour and luminance contrast, respectively involving the luminance sensitive pathways (M-pathways), and the colour sensitive pathways (P-pathways) of the visual system (e.g. Hubel and Livingstone, 1990). The psychophysical data on spatial facilitation point toward a possibly predominant role of the luminance sensitive pathways as an explanation for effects observed with orientation stimuli defined by luminance contrast, and a possibly predominant role of the colour sensitive pathways as an explanation for effects found with context stimuli defined by colour contrast. The spatial dynamics of these two types of mechanisms, as reflected by effects of targetinducer separation, size, or contrast intensity for example, may differ and are to be compared in further psychophysical experiments.

\section{Long-range detection facilitation and practice effects}

Another striking aspect of psychophysically measured spatial interactions is that they often give rise to quite substantial practice effects. Practice effects generally describe the fact that the observer's performance in the psychophysical task gets noticeably better with training, which may consist of a more or less substantial number of trial blocks. Some of the ecological aspects of training, or visual experience, have been emphasized by Polat and Sagi (1994b) within a connectionist approach to long range perceptual interactions. Dorais and Sagi (1997) have reported practice effects on detection performances in contrast masking experiments with contour structures defined by oriented stimuli with a gaussian envelope (Gabor patches). These effects are discussed on the basis of habituative mechanisms in the non-linear filter model used to explain data on spatial interactions in contrast vision. The practice effects here were described as an 'unmasking phenomenon', since the observers' detection performances showed a noticeably diminished sensitivity to the presence of the masking stimuli after training.

In some of the studies with line targets and collinear inducers (e.g. Dresp and Grossberg, 1997), it has been necessary to run subjects in several thousands of training trials with the inducing contours before the lowest possible detection threshold was obtained. Interestingly, in the control conditions where the line target is presented without contour inducers, detection performances evolve only very little, if at all. An account for some of these training effects in spatial facilitation is given in a recent paper (Dresp, 1998) where the evolution of detection thresholds for a line target embedded in a context representing a collinear structure is compared to the evolution of detection performances without collinear structure. The practice effect with the collinear structure is reflected by a decrease in line detection thresholds, and by a systematic organization in the response times with 
the progression of daily trial blocks over a period of one month with two observers. The most likely explanation for practice effects in spatially induced detection facilitation appears to be some progressive reinforcement of interactions between visual mechanisms activated by the target and visual mechanisms activated by the context, combined with an optimization of decision strategies. This view is by and large consistent with Dorais' and Sagi's interpretation of their 'unmasking' effects with practice, which are seen as an interference reduction process at the interface between primary visual mechanisms and higher processes. Further research on practice effects will progressively disentangle the intricate relationship between apparently highly specific structural and temporal factors in long range perceptual interactions.

\section{FROM-SHORT-TO-LONG-RANGE CORTICAL INTERACTIONS AS AN EXPLANATION FOR SPATIAL FACILITATION AND VISUAL GROUPING?}

Referring to neurophysiological evidence for a from-simple-to-complex-cells processing hierarchy in the visual cortex (e.g. Gilbert and Wiesel, 1985), Dresp and Grossberg (1997) have discussed the possible role of interactions involving mechanisms where oriented contrasts carrying the same sign are coded selectively within a narrowly limited spatial scale at a first stage of processing (shortrange mechanisms) and then, at a second stage, are integrated in a more global representation of contour or form by mechanisms that disregard the sign of contrast and operate over a larger spatial scale (long-range mechanisms). It appears that the idea of two distinct stages of cortical processing successfully accounts for some of the specific effects of the relative position, size, spacing, contrast polarity, and maybe to some extent also contrast intensity, of targets and inducers.

The short-range mechanisms of the first processing stage are identified with some of the functional characteristics of cortical simple cells, the long-range mechanisms of the second stage are identified with processing characteristics of cortical complex cells or, more specifically, so-called bipole operators. A focussed account for the extent to which effects of spatial facilitation or suppression may be explained by some of the dynamic properties of Grossberg and Mingolla's cortical network model is given along the following lines.

\section{Collinear facilitation and orthogonal suppression}

Considering that receptive fields of cortical neurons are largely overlapping, sometimes completely superimposed, on the retina, it becomes clear that cortical detectors must interact to enable the visual system to detect regularites and structure in the stimuli, to sort out what belongs where, and to build up a coherent representation of the visual scene as a whole. Spatial facilitation effects produced by stimuli representing collinear structures may involve interactions between cortical detectors with overlapping or spatially adjacent receptive fields, selective to the same orientation (e.g. Dresp, 1993; Kapadia et al., 1995). The ability of the visual system to 
accurately compute the likelihood that spatially separated line fragments belong to the same object contour, as demonstrated in the experiments by Field et al. (1993) mentioned in the introduction, may result from the fact that the firing level of detectors is raised because each of them receives excitatory input not only from the external trigger stimulus, but also from detectors selective to the same orientation, and co-activated by nearby stimuli. This interpretation is consistent with the behavior of visual neurons in Vl of the awake monkey in response to collinear line stimuli (Kapadia et al., 1995). Both the short-range and the long-range contour detection mechanisms in the model by Grossberg and Mingolla operate on the basis of cooperative, or competitive interactions between co-activated detectors.

Observations showing that the detection of a line target is suppressed by a nearby line with orthogonal orientation (e.g. Dresp, 1993; Kapadia et al., 1995) are explained in the model by inhibitory interactions between co-activated detectors selective to different orientations. In this case, the firing level of detectors with overlapping receptive fields is not raised but reduced by the interactions because each of them receives suppressive input from co-activated detectors selective to a different, competing orientation. Again, the interpretation is consistent with responses of visual neurons in VI of the awake monkey to spatially separated, orthogonal lines (Kapadia et al., 1995), and also with the more general evidence for cross-orientation inhibition in the visual cortex (see, for example, Burr et al., 1981 ).

\section{Selectivity to contrast polarity within tight spatial limits}

The sensitivity of spatial facilitation effects to relatively short inducing stimuli, and their selectivity to contrast polarity at precise spatial positions when the separation between targets and inducers is small (e.g. Dresp, 1993; Morgan and Dresp, 1995; Yu and Levi, 1997a), leads to identify the underlying mechanisms with some of the functional characteristics of cortical simple cells. Since these mechanisms operate within clearly limited spatial boundaries, they are referred to as short-range mechanisms here to distinguish them from mechanisms of spatial facilitation operating well beyond these limits, and exhibiting different functional characteristics. These will be discussed in the next subsection.

Short-range interactions between simple cells may be necessary at a first step of processing in visual grouping to filter contrast information of the same sign that has to be organized according to certain rules imposed by the stimulus geometry. Yu and Levi's (1997a) data showing spatial facilitation effects with short inducing lines and targets across short distances and how they can be simulated by the functional characteristics of simple cells with end-stopped receptive fields, illustrate this quite well. By concurrently stimulating the receptive field of a spatial mechanism in the center and in the end-zones or flanks with small contrast stimuli of opposite sign in all possible combinations, they find facilitatory and inhibitory effects similar to those reported with Gabor patches by Polat and Sagi (1993, 1994a, b). The spatial dynamics reflected by their observations are well suited to simulate a cortical 
mechanism that enables the brain to detect structure in some regular textures or periodic patterns where it is important that individual. equally spaced segments perceptually stand out as a group against a non-structured background (Yu and Levi, 1997a). Yu and Levi's end-stopping model as an explanation for short-range spatial facilitation is consistent with the neurophysiological evidence for end-stopping in the visual cortex reported in Hubel and Wiesel's (e.g. 1968) classic studies on receptive field properties of cortical neurons. Although spatial interactions in contrast detection revealing center-surround antagonism in the receptive field of the underlying mechanism has some time ago been identified with the functional properties of retinal cells (Westheimer, 1965, 1967), it is now a well-established working hypothesis that spatial facilitation results mainly from cortical interactions.

\section{Facilitation across larger separations and contrast signs}

When rather long lines or edges are used as inducers and targets, spatial facilitation occurs at target inducer separations well beyond the limits of the short-range interactions, regardless of the contrast sign carried by either the target or the inducer (Zenger and Sagi, 1996; Dresp and Grossberg, 1997; Wehrhahn and Dresp, 1998). Mechanisms based on the functional properties of simple cells, with or without end-stopping properties, are insufficient to account for spatial facilitation under those conditions. It is particularly difficult to see how simple cell mechanisms would account for the fact that these long-range facilitation effects are generally stronger with high contrast inducers (e.g. Zenger and Sagi, 1996; Wehrhahn and Dresp, 1998), given that the latter produce masking, or suppression, within the short-range spatial scale. The fact that long-range spatial facilitation, which describes effects obtained with longer targets and inducers separated by larger gaps, gets stronger when the inducer contrast increases seems to indicate that increased contrast strength compensates for decreased stimulus proximity within the perceptive field reflected by the psychophysical observations. Where could the underlying mechanisms be located in the visual cortex, and which kind of interactions would they involve?

Some plausible candidate hypotheses for long-range contour integration across contrast polarities by cortical mechanisms sensitive to intensity but not to polarity of contrast are suggested in Grossberg and Mingolla's model. On the basis of psychophysical observations on detection facilitation, Dresp and Grossberg (1997) have discussed some of the functional properties of these mechanisms. They are generally identified with the functional properties of cortical complex cells, but possess the additional characterstic of so-called bipole operators (Grossberg and Mingolla, 1985), which means that their receptive field is described by two large, adjacent regions defining a bow-tie shaped surface. The optimal stimulus for such a receptive field are two collinear lines of any contrast polarity.

Complex cortical neurons are often described as having much larger receptive fields than simple cells, and have been found to be sensitive to the strength of contrast, but not to its sign (e.g. Hubel and Wiesel, 1968). Furthermore, there is 
neurophysiological evidence that cortical cells with response properties identified with the functional characteristics of bipole operators participate in the detection of illusory contours and apparent lines which perceptually bridge the spatial gaps between contrast lines or edges (Von der Heydt et al., 1984). It is conceivable that complex cell mechanisms define a higher stage of cortical processing in visual grouping. This higher level of processing would become critically activated whenever mechanisms with higher tolerance for spatial separation are required, and the stimulus or visual scene exhibits non-periodic variations in contrast sign and/or intensity, like many complex patterns and textures. In general, interactions between detectors with a bipole receptive field structure provide a plausible explanation for spatial facilitation with relatively long line targets and collinear inducing lines (Wehrhahn and Dresp, 1998), or with long, widely spaced edges across gaps up to 2.5 deg of visual angle (Dresp and Grossberg, 1997).

Some of the evidence from spatial facilitation experiments suggests an organization in the different mechanisms according to some kind of from-simple-tocomplex-cells processing hierarchy. The idea of such a hierarchy, which also clarifies why there is a functional separation between short-range and long-range effects in the first place, provides a ready account for the fact that the detection of bi-sected target lines is only facilitated when each half of the line carries the same contrast sign as the nearest inducing line or edge (Dresp and Grossberg, 1997). This finding suggests that the local filtering of spatial information carrying the same sign precedes the processing of global structure. The from-short-to-long-range processing hierarchy hypothesis is largely consistent with neurophysiological evidence for intrinsic connectivity between detectors with distinct functional properties in the visual cortex (Gilbert and Wiesel, 1985). It is one of the many key hypotheses in Grossberg and Mingolla's original model, and more recent versions which include the FAÇADE theory (Grossberg, 1994).

\section{REFERENCES}

Burr D., Morrone, C. and Maffei, L. (1981). Intra-cortical inhibition prevents simple cells from responding to textured visual patterns, Experimental Brain Research 43, 455-458.

Dorais, A. and Sagi. D. (1997). Contrast masking effects change with practice. Vision Resectrch 37, $1725-1734$.

Dresp, B. (1991). Etude psychophysique des mécanismes et processus de structuration dans la perception des formes, Unpublished Doctoral Thesis, Université Paris V. France.

Dresp, B. (1993). Bright lines and edges facilitate the detection of small light targets. Spatial Vision 7, 213-225.

Dresp, B. (1998). The effect of practice on the visual detection of near-threshold lines, Spatial Vision 11, 315-327.

Dresp. B. and Bonnet. C. (1991). Psychophysical evidence for low-level processing of illusory contours and surfaces in the Kanizsa square, Vision Research 31, 1813-1817.

Dresp, B. and Bonnet, C. (1993). Psychophysical measures of illusory form perception: further evidence for local mechanisms, Vision Research 33. 759-766. 
Dresp, B. and Bonnet, C. (1995). Subthreshold summation with illusory contours, Vision Research 35, $1071-1078$.

Dresp. B. and Grossberg, S. (1997). Contour integration across polarities and spatial gaps: From contrast filtering to bipole cooperation, Vision Research 37, 913-924.

Dresp, B. and Grossberg, S. (1998). Spatial facilitation by color and luminance edges: boundary, surface, and attentional factors, Vision Research (in revision, conditionally accepted for publication).

Dresp, B., Breitmeyer, B. and Bonnet, C. (1998). Contrast detection and positional coding of collinear lines, Perception (Suppl. ECVP abstract) 27, 7.

Field, D. J., Hayes, A. and Hess, R. F. (1993). Contour integration by the human visual system: Evidence for a local 'association field', Vision Research 33, 173-193.

Foley, J. M. and Legge, G. E. (1981). Contrast detection and near-threshold discrimination in human vision, Vision Research 21, 1041-1053.

Gilbert, C. D. and Wiesel, T. N. (1985). Intrinsic connectivity and receptive field properties in visual cortex, Vision Research 25, 365-374.

Gilbert, C. D. and Wiesel, T. N. (1990). The influence of contextual stimuli on the orientation selectivity of cells in the primary visual cortex of the cat, Vision Research 30, 1689-1701.

Grossberg, S. (1994). 3-D vision and figure-ground separation by visual cortex, Perception and Psychophysics 55, 48-120.

Grossberg, S. and Mingolla, E. (1985). Neural dynamics of perceptual grouping: textures, boundaries, and emergent segmentations, Perception and Psychophysics 38, 141-171.

Hubel, D. H. and Wiesel, T. N. (1965). Receptive fields and functional architcture in two nonstriate visual areas (18 and 19) of the cat, Journal of Neurophysiology 28, 229-289.

Hubel, D. H. and Wiesel, T. N. (1968). Receptive field and functional architecture of monkey striate cortex, Journal of Physiology 195, 215-243.

Hubel, D. H. and Livingstone, M. S. (1990). Color and contrast sensitivity in the lateral geniculate body and primary visual cortex of the macaque monkey, Journal of Neuroscience 10, 2223-2237.

Jung, R. and Spillmann, L. (1970). Receptive-field estimation and perceptive integration in human vision, in: Early Experience and Visual Information Processing in Perceptual and Reading Disorders, F. A. Young and D. B. Lindslay (Eds), pp. 181-197. National Academy of Sciences, Washington.

Kapadia, M. K., Ito, M., Gilbert, C. D. and Westheimer, G. (1995). Improvement in visual sensitivity by changes in local context: parallel studies in human observers and in V1 of alert monkeys, Neuron 15, 843-856.

Kovács, I. and Julesz, B. (1993). A closed curve is much more than an incomplete one: Effect of closure in completion of segmented contours, Proceedings of the National Academy of Science, USA 90, 7495-7497.

Leonards, U. and Singer, W. (1998). Two segmentation mechanisms with differential sensitivity for colour and luminance contrast, Vision Research 38, 101-109.

Morgan, M. J. and Dresp, B. (1995). Contrast detection facilitation by spatially separated targets and inducers, Vision Research 35, 1019-1024.

Polat, U. and Sagi, D. (1993). Lateral interactions between spatial channels: Suppression and facilitation revealed by lateral masking experiments, Vision Research 33, 993-999.

Polat, U. and Sagi, D. (1994a). The architecture of perceptual spatial interactions, Vision Research 28 , $115-132$.

Polat, U. and Sagi, D. (1994b). Spatial interactions in human vision: from near to far via experience dependent cascades of connections, Proceedings of the National Academy of Science, USA 91, $1206-1209$.

Von der Heydt, R.. Peterhans, E. and Baumgartner, G. (1984). Illusory contours and cortical neuron responses, Science 224, 1260-1262.

Wehrhahn, C. and Dresp, B. (1998). Detection facilitation by collinear stimuli in humans: Dependence on strength and sign of contrast, Vision Research 38, 423-428. 
Westheimer, G. (1965). Spatial interacton in the human retina during scotopic vision, Journal of Physiology 181, 812-894.

Westheimer, G. (1967). Spatial interaction in human cone vision, Journal of Physiology 190, $139-154$.

Westheimer, G. (1979). The spatial sense of the eye, Investigative Ophthalmology and Visual Science 17, 893-912.

Yu, C. and Levi, D. M. (1997a). Spatial facilitation predicted with end-stopped spatial filters, Vision Research 37, 3117-3127.

Yu, C. and Levi, D. M. (1997b). Cortical end-stopped perceptive fields: evidence from dichoptic and amblyopic studies, Vision Research 37, 2261-2270.

Zenger, B. and Sagi, D. (1996). Isolating excitatory and inhibitory nonlinear spatial interactions involved in contrast detection, Vision Research 36, 2497-2513.

Zucker S. W. and Davis, S. (1988). Points and end-points: a size/spacing constraint for dot grouping, Perception 17, 229-247. 